\title{
New sampling expansions for bandlimited signals based on chromatic derivatives
}

\author{
P. P. Vaidyanathan ${ }^{\diamond}$, A. Ignjatovic ${ }^{\ddagger}$, and M. J. Narasimha ${ }^{\dagger}$, \\ $\diamond$ Dept. Electrical Engr., Caltech. Pasadena, CA; ppvnath@systems.caltech.edu \\ $\ddagger$ Kromos Technology, Inc.; aleks@kromos.com * \\ †Stanford University and Comstellar Technologies; sim@nova.stanford.edu
}

\begin{abstract}
Shannon's sampling theorem for bandlimited signals has been generalized in many directions in the last few decades. These extensions lead to various types of signal representations having different sets of basis functions. One particular extension proposed by Papoulis and later developed further by Brown can be interpreted in terms of a continuous time minimally sampled filter bank. In this paper we take a second look at these filter banks and use a continuous time version of the familiar biorthogonality property to obtain further insights into these sampling theorems. This viewpoint also makes a natural connection to the theory of orthogonal polynomials. We then elaborate on an elegant representation called the chromatic derivative expansion based on the use of Chebyshev polynomials. Using this expansion, the analysis/synthesis system can be described with a Chebyshev/Bessel pair of functions.
\end{abstract}

\section{INTRODUCTION}

The uniform sampling theorem for bandlimited signals due to Shannon and Nyquist has been generalized in many directions in the last six decades [6]. These extensions lead to various types of signal representations having different sets of basis functions. One particular extension proposed by Papoulis [10] and later developed further by Brown [1] can be interpreted in terms of a continuous time minimally sampled filter bank. This extension brings out the derivative sampling theorem as a special case.

In this paper we take a second look at continuous time filter banks and use a continuous time version of the familiar biorthogonality property [14], [15], [16] to obtain further insights into these continuous-time sampling theorems. The infinite channel filter bank, in particular, leads to some very useful insights, and makes a connection between orthogonal polynomial theory and filter bank basis.

After a review of these ideas, we elaborate on an especially elegant representation called the chromatic derivative expansion, advanced by Ignjatovic in several internal reports of Kromos technology [4], [5]. This is based on the use of orthogonal polynomials in the frequency do. main, and corresponding time functions that are defined by these polynomials and their weight functions. In particular we consider the Chebyshev/Bessel pair of functions and demonstrate that they yield an analysis/synthesis filter bank with the PR property.

*Kromos Technology, Inc., is a subsidiary of Comstellar Technologies.

\section{CONTINUOUS TIME FILTER BANKS}

Given a continuous time $\pi$-BL signal signal $x(t)$, that is, a signal bandlimited to $-\pi<\omega<\pi$, we know that it can be recovered from the uniformly spaced samples $x(n)$. These samples are spaced apart by one second (i.e., sampling rate $=1 \mathrm{~Hz}$ ). It is well-known that if the signal and its $M-$ 1 derivatives are available for sampling, then these can be sampled at $M$ times smaller rate, and the signal $x(t)$ recovered from these samples.

To generalize these ideas further, consider Fig. 1 where the signal $x(t)$ is split into $M$ signals using the analysis filters $H_{k}(\omega)$. The filter outputs are then sampled at the rate $1 / M \mathrm{~Hz}$ (samples spaced apart by $M$ ). The figure also shows a synthesis bank where the $k$ th synthesis filter is driven by the impulse train obtained from sampling $v_{k}(t)$. The outputs of the synthesis filters are added together to get the reconstructed signal $\widehat{x}(t)$. Given the analysis filters $H_{k}(\omega)$, if we can solve for the synthesis filters $F_{k}(\omega)$ such that $x(t)=x(t)$, then the filter bank has perfect reconstruction $(P R)$. This leads to the observation that every $P R$ filter bank has associated with it a "sampling theorem."

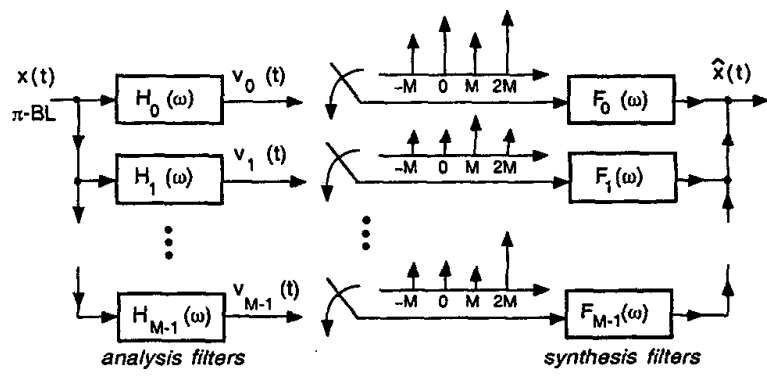

Figure 1. A continuous-time $M$-band filter bank.

For example, if we choose

$$
H_{k}(\omega)=(j \omega)^{k}
$$

then the $k$ th analysis filter performs $k$ th order differentiation of the input $x(t)$. In this case we get the derivative sampling theorem. As another example suppose $H_{k}(\omega)$ and $F_{m}(\omega)$ are derived from a standard digital filter bank 
$\left\{H_{d, k}(z), F_{d, m}(z)\right\}$ according to the relation

$$
H_{k}(\omega)= \begin{cases}H_{d, k}\left(e^{j \omega}\right) & -\pi<\omega<\pi \\ 0 & \text { otherwise }\end{cases}
$$

and similarly for $F_{k}(\omega)$. If the digital filter bank has the PR or biorthogonality property [15], then it can readily be verified that the system of Fig. 1 also has the PR property. This is a simple way to generate an infinity of examples, because there are unlimited examples of digital PR filter banks. Before proceeding further we review the idea of biorthogonality in the context of continuous time filter banks.

\section{BIORTHOGONALITY}

The derivation of special cases such as the derivative sampling theorems based on the filter bank formulation of Fig. 1 is insightful but rather elaborate [10]. The synthesis filters were derived by formulating alias components and cancelling them by explicit frequency domain constructions of the functions $F_{k}(\omega)$. At the time of their development, the idea of biorthogonality was not well known in the signal processing community. The details of Papoulis's construction can be found in [10], and are also reviewed in Problem 5.13 of [15]. The resulting filters are not practically implementable filters. Nevertheless, the construction provides the theory for derivative sampling.

In this section we formulate the biorthogonality property and show how it directly leads to perfect reconstruction. The output signal in Fig. 1 can be expressed as

$$
\widehat{x}(t)=\sum_{k=0}^{M-1} \sum_{n=-\infty}^{\infty} v_{k}(M n) f_{k}(t-n M)
$$

Thus any signal which can be represented as the output of this filter bank belongs to the space $\mathcal{S}$ spanned by the doubly indexed set of functions

$$
\eta_{k, n}(t)=f_{k}(t-n M), 0 \leq k \leq M-1,-\infty \leq n \leq \infty
$$

We shall assume that $\eta_{k, n}(t)$ are linearly independent. Suppose the input $x(t)$ itself belongs to the space $\mathcal{S}$. Then it can be expressed in the form

$$
x(t)=\sum_{k=0}^{M-1} \sum_{n=-\infty}^{\infty} a_{k, n} f_{k}(t-n M)
$$

for some set of constants $a_{k, n}$. Equivalently

$$
X(\omega)=\sum_{k=0}^{M-1} \sum_{n=-\infty}^{\infty} a_{k, n} F_{k}(\omega) e^{-j \omega M n}
$$

For such an input, we show that the perfect reconstruction property $\widehat{x}(t)=x(t)$ is satisfied if the filters satisfy the biorthogonality property defined as follows:

$$
\frac{1}{2 \pi} \int_{-\pi}^{\pi} H_{k}(\omega) F_{m}(\omega) e^{j \omega M n} d \omega=\delta(k-m) \delta(n)
$$

where $\delta($.$) represents the Kronecker delta function.$

Proof. The sample $v_{m}(M \ell)$ of the $m$ th subband signal is the inverse Fourier transform of $H_{m}(\omega) X(\omega)$ evaluated at $t=M \ell$ for integers $\ell$. Thus

$$
\begin{aligned}
& v_{m}(M \ell)=\int_{-\pi}^{\pi} H_{m}(\omega) X(\omega) e^{j \omega M \ell} \frac{d \omega}{2 \pi} \\
= & \sum_{k=0}^{M-1} \sum_{n=-\infty}^{\infty} a_{k, n} \int_{-\pi}^{\pi} H_{m}(\omega) F_{k}(\omega) e^{j \omega M(\ell-n)} \frac{d \omega}{2 \pi} \\
= & \sum_{k=0}^{M-1} \sum_{n=-\infty}^{\infty} a_{k, n} \delta(k-m) \delta(\ell-n)
\end{aligned}
$$

where the last equality follows from biorthogonality (5). This shows that

$$
v_{m}(M \ell)=a_{m, \ell}
$$

Using (1) we see that the reconstructed signal is

$$
\begin{aligned}
\widehat{x}(t) & =\sum_{k=0}^{M-1} \sum_{n=-\infty}^{\infty} v_{k}(M n) f_{k}(t-n M) \\
& =\sum_{k=0}^{M-1} \sum_{n=-\infty}^{\infty} a_{k, n} f_{k}(t-n M) \\
& =x(t)
\end{aligned}
$$

where the last equality follows from (3). This proves that biorthogonality implies perfect reconstruction. $\quad \nabla \nabla \nabla$

\section{INFINITE BAND FILTER BANKS}

Consider Fig. 2 where a $\pi$-BL signal $x(t)$ is passed through a filter bank with an infinite number of channels. The outputs of the analysis filters are sampled only at one point, namely $t=0$. This system can therefore be regarded as a special case where $M \rightarrow \infty$. The biorthogonality condition for this filter bank cannot be derived readily from (5) because the limiting process $M \rightarrow \infty$ is tricky. For this system we define biorthogonality as follows:

$$
\int_{-\pi}^{\pi} F_{k}(\omega) H_{m}(\omega) \frac{d \omega}{2 \pi}=\delta(k-m), 0 \leq k, m \leq \infty
$$

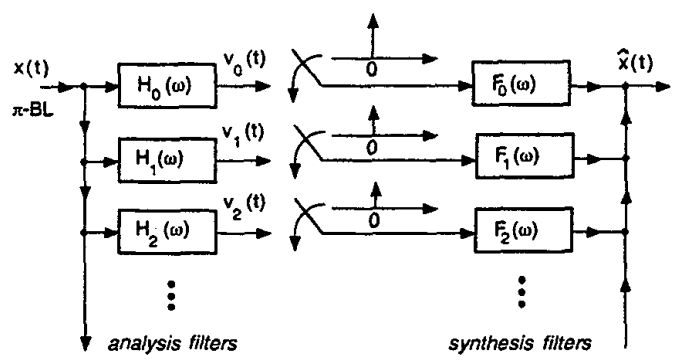

Figure 2. A continuous-time infinite-band filter bank. 
Assume as in Sec. III that the input $x(t)$ is in the space spanned by the synthesis filters $F_{k}(\omega)$, that is,

$$
\boldsymbol{x}(t)=\sum_{k=0}^{\infty} c_{k} f_{k}(t)
$$

or equivalently $X(\omega)=\sum_{k=0}^{\infty} c_{k} F_{k}(\omega)$. We can then show that the biorthogonality property implies perfect reconstruction:

Proof. The output $v_{m}(t)$ sampled at $t=0$ is given by

$$
\begin{aligned}
v_{m}(0) & =\int_{-\pi}^{\pi} X(\omega) H_{m}(\omega) \frac{d \omega}{2 \pi} \\
& =\sum_{k=0}^{\infty} c_{k} \int_{-\pi}^{\pi} F_{k}(\omega) H_{m}(\omega) \frac{d \omega}{2 \pi}=c_{m}
\end{aligned}
$$

where the last equality follows from biorthogonality (6). It then follows that the reconstructed signal is

$$
\begin{aligned}
\widehat{X}(\omega) & =\sum_{m=0}^{\infty} v_{m}(0) F_{m}(\omega) \\
& =\sum_{m=0}^{\infty} c_{m} F_{m}(\omega)=X(\omega)
\end{aligned}
$$

This proves that biorthogonality (6) implies perfect reconstruction.

$\nabla \nabla \nabla$

For the case of finite $M$, we explained at the end of Sec. II how to construct examples of continuous time biorthogonal filter banks by starting from examples of digital filter banks. For infinite $M$ such constructions do not work because digital filter banks always have finite $M$. However, by using known results from mathematics it is indeed possible to construct many examples. One such construction results if the analysis filters are differentiators $H_{k}(\omega)=(j \omega)^{k}$. In this case

$$
v_{k}(0)=x^{(k)}(0)
$$

that is, the $k$ th derivative of $x(t)$ evaluated at $t=0$. By writing the standard Taylor series for $x(t)$ around $t=0$

$$
x(t)=\sum_{k=0}^{\infty} x^{(k)}(0) \frac{t^{k}}{k !}
$$

we see that if the synthesis filter impulse responses are chosen as $f_{k}(t)=t^{k} / k !$, then we have perfect reconstruction. The analysis filter responses $H_{k}(\omega)=(j \omega)^{k}$ appear to be unbounded but they can be restricted to be zero outside the range $-\pi<\omega<\pi$ because the input is bandlimited anyway. The synthesis responses, which are unbounded in time, clearly result in impractical filters unless we restrict the reconstruction to be over a finite window in time. The approximation error due to truncation of the infinite series grows very noticeably as the reconstruction window increases in duration [5].

\section{ORTHOGONAL POLYNOMIALS}

The Taylor series expansion is not the only example that can be derived from the infinite band filter bank. Many other impressive examples can be found based on the theory of orthogonal polynomials [3]. A set of polynomials $P_{k}(x)$ is said to be orthonormal on the interval $[-1,1]$ with respect to a weighting function $v(x)$ if

$$
\int_{-1}^{1} P_{k}(x) P_{m}(x) v(x) d x=\delta(k-m)
$$

for $0 \leq k, m \leq \infty$. Suppose we define a set of filters based on these polynomials as follows:

$$
\begin{aligned}
H_{k}(\omega) & =\sqrt{2} v(\omega / \pi) P_{k}(\omega / \pi) \Pi(\omega) \\
F_{k}(\omega) & =\sqrt{2} P_{k}(\omega / \pi) \Pi(\omega)
\end{aligned}
$$

where $\Pi(\omega)$ is the bandlimiting function

$$
\Pi(\omega)= \begin{cases}1 & \text { for }|\omega|<\pi \\ 0 & \text { otherwise }\end{cases}
$$

Then the orthogonality property (7) translates to the biorthogonality property (6)! Thus, given any set of orthogonal polynomials $\left\{P_{k}(x)\right\}$, we can always associate with it an infinite band filter bank with the biorthogonality property. ${ }^{1}$ An interesting example is offered by the beautiful family of Chebyshev polynomials as described next.

\section{CHROMATIC DERIVATIVES}

The role of Chebyshev polynomials in the context of partial reconstruction was first introduced by Ignjatovic in a series of internal reports from Kromos Technology [4], [5]. Chebyshev polynomials $T_{k}(x)$ are defined by the recursion

$$
T_{N+1}(x)=2 x T_{N}(x)-T_{N-1}(x), N \geq 1,
$$

with $T_{0}(x)=1$, and $T_{1}(x)=x$. For example, the recursion leads to

$$
T_{2}(x)=2 x^{2}-1, \quad T_{3}(x)=4 x^{3}-3 x \ldots
$$

and so forth. For $-1 \leq x \leq 1$ the Chebyshev polynomial can be written as $\widehat{T}_{N}(x)=\cos \left(N \cos ^{-1}(x)\right)$ which shows that $T_{N}(x)$ oscillates between -1 and 1 in the interval $[-1,1]$. These polynomials satisfy the orthogonality relation [3]

$$
\int_{-1}^{1} \frac{T_{k}(x) T_{m}(x)}{\sqrt{1-x^{2}}} d x= \begin{cases}0 & \text { for } k \neq m \\ \pi / 2 & \text { for } k=m \neq 0 \\ \pi & \text { for } k=m=0\end{cases}
$$

That is, they are orthogonal in $[-1,1]$ with weight function $v(x)=1 / \sqrt{1-x^{2}}$.

\footnotetext{
The connection between orthogonal polynomials and filter banks was also noticed in the report by Cushman [2], also from Kromos Technology.
} 


\section{VI.1. Chromatic Derivative Filter Banks}

It is easy to see that a frequency response represented by a polynomial in $\omega$ corresponds to a linear combination of differential operators in the time domain. Filters based on such combinations were called chromatic derivative operators [5]. It has been shown that the expansion of a signal using chromatic derivatives gives an attractive alternative to Taylor series expansion. For example the truncated version of chromatic expansion has the local approximation property similar to Taylor expansion, but unlike the truncated Taylor expansion, the error grows much more gracefully as we move away from the point of expansion [5]. The analysis filters in the chromatic filter bank are defined as

$$
H_{k}(\omega)=j^{k} T_{k}(\omega / \pi)
$$

and the synthesis filters chosen as

$$
F_{m}(\omega)= \begin{cases}2 W(\omega) H_{m}^{*}(\omega) & \text { for } m \neq 0 \\ W(\omega) H_{m}^{*}(\omega) & \text { for } m=0\end{cases}
$$

where the weighting function $W(\omega)$ is given by

$$
W(\omega)=\frac{2 \Pi(\omega)}{\pi \sqrt{1-(\omega / \pi)^{2}}}
$$

Note that filter responses are zero for $|\omega|>\pi$. From the orthogonality property (8) it follows that

$$
\int_{-\pi}^{\pi} W(\omega) T_{k}(\omega / \pi) T_{m}(\omega / \pi) \frac{d \omega}{2 \pi}= \begin{cases}0 & k \neq m \\ 0.5 & k=m \neq 0 \\ 1 & k=m=0\end{cases}
$$

The filter bank shown in Fig. 2 therefore has the PR property. The relevance of the factor $j^{k}$ comes from the fact that $T_{k}(\omega / \pi)$ is an even function of $\omega$ for even $k$ and odd function of $\omega$ for odd $k$. Thus, with the help of the factor $j^{k}$, the impulse response $h_{k}(t)$ is guaranteed to be real for all $k$. Using properties of Fourier transforms, it also follows that $h_{k}(t)$ is itself even for even $k$ and odd for odd $k$.
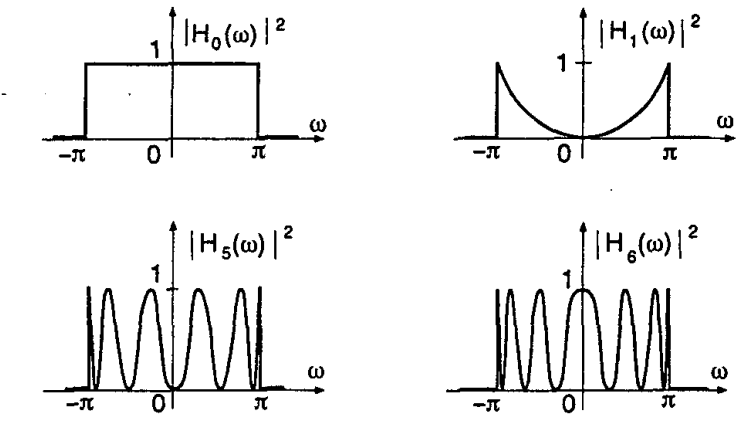

Figure 3. Examples of analysis filters in the Chebyshevbased analysis bank. The magnitude square response $\left|H_{k}(\omega)\right|^{2}$ is shown for various values of $k$.
Figure 3 shows the responses $\left|H_{k}(\omega)\right|^{2}$ for some of the analysis filters, and Fig. 4 shows some of the synthesis filters. The analysis filters have zeros distributed in the interval $-\pi<\omega<\pi$, and the zeros of any two analysis filters are disjoint. The synthesis filters in the Chebyshev filter bank have the factor $W(\omega)$ which makes them grow in an unbounded manner as $\omega$ approaches $\pm \pi$ (Fig. 4). Notice that all the filters are discontinuous at $\omega= \pm \pi$ because they are bandlimited.
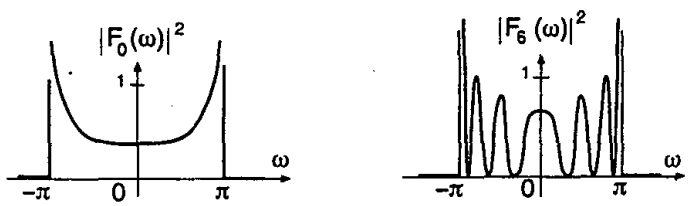

Figure 4. Magnitude square response $\left|F_{k}(\omega)\right|^{2}$ of two of the synthesis filters in the Chebyshev-based filter bank.

It has been shown by Ignjatovic [5] that the synthesis bank (10) can be expressed in a elegant form using Bessel functions. The chromatic analysis/synthesis system can therefore be regarded as the Chebyshev/Bessel filter bank. To see this, first recall that the $m$ th order Bessel function $J_{m}(t)$, defined as,

$$
J_{m}(t)=\int_{-\pi}^{\pi} e^{j(m \omega-t \sin \omega)} \frac{d \omega}{2 \pi}
$$

is bandlimited to $|\omega|<1$, and its Fourier transform in $|\omega|<1$ is given by [11]

$$
\widehat{J}_{m}(\omega)=\frac{2(-j)^{m} T_{m}(\omega)}{\sqrt{1-\omega^{2}}}
$$

Using this we can write the synthesis filters as

$$
F_{m}(\omega)= \begin{cases}\frac{2}{\pi} \widehat{J}_{0}(\omega / \pi) H_{m}^{*}(\omega)=\frac{2}{\pi} \widehat{J}_{m}(\omega / \pi) & m \neq 0 \\ \frac{1}{\pi} \widehat{J}_{0}(\omega / \pi) & m=0\end{cases}
$$

VI.2. Orthonormal Chromatic Derivative Filter Banks

The weighting function $W(\omega)$ can be distributed more evenly between the analysis and synthesis filters by choosing the filters as follows:

$$
H_{k}(\omega)= \begin{cases}j^{k} T_{k}(\omega / \pi) \sqrt{2 W(\omega)} & \text { for } k \neq 0 \\ \sqrt{W(\omega)} & \text { for } k=0\end{cases}
$$

and

$$
F_{m}(\omega)=H_{m}^{*}(\omega)
$$

for all $m$. Evidently the biorthogonality property continues to be satisfied. Moreover, all the filters now have unit energy, that is,

$$
\int_{-\pi}^{\pi}\left|H_{m}(\omega)\right|^{2} \frac{d \omega}{2 \pi}=\int_{-\pi}^{\pi}\left|F_{m}(\omega)\right|^{2} \frac{d \omega}{2 \pi}=1
$$


The normalized system is such that the synthesis and analysis filters are both unbounded at $\omega= \pm \pi$, because of the factor $\sqrt{W(\omega)}$. However, they are still square-integrable, and have unit energy. Thus, if the input to each synthesis filter is additive white noise, the noise gain (filter energy) is unity which shows that noise is not unduly amplified even though the filter responses are unbounded at $\omega= \pm \pi$.

\section{CONCLUDING REMARKS}

The merits of chromatic derivatives based on the Chebyshev/Bessel pair have been established in [5]. For the more general case of chromatic filter banks where each $H_{k}(\omega)$ is a polynomail of order $<M$, there is a simple way to obtain the synthesis filters $F_{k}(\omega)$ using a time domain approach. Both conceptually and procedurally this is much simpler than the frequency domain approach of Papoulis [10]. This was first pointed out by Cushman, and the details can be found in $[8]$.

Chebyshev polynomials are optimal in the minimax sense $[9,12,15]$. That is, given any degree- $N$ polynomial $R_{N}(x)$ with the same highest coefficient (coefficient of $x^{N}$ ) as the Chebyshev polynomial, it can be shown that the maximum of $\left|R_{N}(x)\right|$ in $[-1,1]$ is at least as large as the maximum of $\left|T_{N}(x)\right|$ in $[-1,1]$ (which is precisely unity). This is a standard property used to show that Chebyshev filters have minimum passband attenuation among all continuous-time all-pole filters [12]. The Dolph-Chebyshev window, used for FIR filter design and for antenna array design is another example of the application of Chebyshev polynomials. As to how this optimality helps in the context of the filter bank of Fig. 2 remains to be explored.

Many other standard examples of orthogonal polynomials can be found in the mathematics literature [3], [7], [13]. For example the Legendre polynomials defined using Rodrigue's formula

$$
P_{k}(x)=\frac{1}{2^{k} k !} \frac{d^{k}\left[\left(x^{2}-1\right)^{k}\right]}{d x^{k}}, \quad 0 \leq k \leq \infty
$$

are orthogonal in $[-1,1]$ with a weighting function of unity. It can be shown that $P_{2 n}(x)$ is even and $P_{2 n+1}(x)$ is odd, and the subscript is precisely the degree of the polynomial. For example the first few polynomials are $P_{0}(x)=1, P_{1}(x)=x$,

$$
P_{2}(x)=\left(3 x^{2}-1\right) / 2, P_{3}(x)=\left(5 x^{3}-3 x\right) / 2, \quad \ldots
$$

and so forth. Thus, the polynomials are similar to Chebyshev polynomials in some respects. They are not normalized because

$$
\left\|P_{k}\right\|^{2}=2 /(2 k+1)
$$

For convenience define

$$
Q_{k}(x)=P_{k}(x) /\left\|P_{k}\right\| .
$$

Let $f(x)$ be an arbitrary signal defined on $[-1,1]$. It is well-known [3] that the $N$ th order least squares polynomial approximation of $f(x)$ has the form

$$
\widehat{f}(x)=\sum_{k=0}^{N} b_{k} Q_{k}(x)
$$

where $b_{k}=\int_{-1}^{1} f(x) Q_{k}(x) d x$. In terms of the filter bank system given in Fig. 2 this optimality means that if we retain the subband samples for the first $N+1$ channels only, then the partial reconstruction $\widehat{X}(\omega)$ is the best $N$ th order polynomial approximation of $X(\omega)$ in $[-\pi, \pi]$.

It is clear that many examples of the infinite band filter bank can be derived by starting from examples in the mathematics literature. As to how these might help in practical applications remains to be explored.

\section{REFERENCES}

[1] Brown, Jr., J. L. "Multi-channel sampling of lowpass signals," IEEE Trans. Circuits and Systems, pp. 101-106, Feb. 1981 .

[2] Cushman, M. "Image compression", Tech. report, Kromos Technology, March, 2001 (http://www.kromos.com).

[3] Hilderbrand, F. B. Introduction to numerical analysis, Dover Publications, Inc., New York, 1987.

[4] Ignjatovic, A. "Introduction to signal processing based on differential operators", Technical report, Kromos Technology, February, 2001 (http://www.kromos.com).

[5] Ignjatovic, A. "Numerical differentiation and signal processing", Technical report, Kromos Technology, February, 2001 (http://www.kromos.com).

[6] Jerri, A. J. "The Shannon sampling theorem - its various extensions and applications: a tutorial review," Proc. of the IEEE, pp. 1565-1596, Nov. 1977.

[7] Kreyszig, E. Advanced Engineering Mathematics, John Wiley, and Sons, Inc., 1972.

[8] Narasimha, M. J., Cushman, M., and Vaidyanathan, P. P. "Finite band chromatic derivative filter banks", in preparation.

[9] Oppenheim, A. V., Schafer, R. W., and Buck, J. R. Discrete-time signal processing, Prentice Hall, Inc., 1999.

[10] Papoulis, A. "Generalized sampling expansion", IEEE Trans. Circuits and Systems, pp. 652-654, Nov. 1977.

[11] Papoulis, A. Systems and transforms with applications in optics, McGraw Hill, Inc. 1968.

[12] Sedra, A. S., and Brackett, P. O Filter theory and design: active and passive, Matrix Publishers, Inc., Beaverton, OR, 1978.

[13] Szego, O. Orthogonal polynomials, American Mathematical Society, Providence, RI, 1967:

[14] Vaidyanathan, P. P., and Vrcelj, B. "Biorthogonal partners and applications," IEEE Trans. Signal Processing, vol. 49, pp. 1013-1027, May 2001.

[15] Vaidyanathan, P. P. Multirate systems and filter banks, Prentice Hall, Inc., 1993.

[16] Vetterli, M., and Kovačević, J. Wavelets and subband coding, Prentice Hall, Inc., 1995. 